\title{
Aspect Ratio
}

National Cancer Institute

\section{Source}

National Cancer Institute. Aspect Ratio. NCI Thesaurus. Code C73486.

The ratio between the longest and shortest dimension of an object. 